\title{
Effects of Macamide B on the Expression of SIRT1 in Brain Tissue of Neonatal Mice with Hypoxic-ischemic Brain Damage
}

\author{
Ke Niu ${ }^{1}$, Fengjie Xu ${ }^{1}$, Guangyong Wang ${ }^{2}$, Manhong Zeng ${ }^{3}$, * \\ ${ }^{1}$ School of Clinical Medicine, Guangdong Pharmaceutical University, Guangzhou, People's Republic of China \\ ${ }^{2}$ Department of Forensic Science, Intermediate People's Court of Huangshan City, Huangshan, People's Republic of China \\ ${ }^{3}$ School of Biosciences \& Biopharmaceutics, Guangdong Pharmaceutical University, Guangzhou, People's Republic of China
}

Email address:

Zengmanhong@163.com (Manhong Zeng)

${ }^{*}$ Corresponding author

\section{To cite this article:}

Ke Niu, Fengjie Xu, Guangyong Wang, Manhong Zeng. Effects of Macamide B on the Expression of SIRT1 in Brain Tissue of Neonatal Mice with Hypoxic-ischemic Brain Damage. Biomedical Sciences. Vol. 7, No. 3, 2021, pp. 64-68. doi: 10.11648/j.bs.20210703.11

Received: June 2, 2021; Accepted: June 19, 2021; Published: July 15, 2021

\begin{abstract}
Neonatal hypoxic-ischemic brain damage (HIBD) is the main cause of severe neurological diseases and death in newborns. Macamide B is an effective monomer extracted from Maca (Lepidium meyenii Walpers), which has important biological activities such as neuroprotection and neuromodulation. The purpose of this study was to investigate whether Macamide B can exert neuroprotective effects on HIBD in newborn mice by regulating Silent information regulator factor 2-related enzyme 1 (SIRT1). A modified Rice-Vannucci method was used to construct the HIBD model of newborn mice. The pups were divided into the following groups: sham group, HI group, and Macamide B group. On the first and third days after hypoxic-ischemic (HI), immunofluorescence and Western blot experiments were used to detect the expression level of SIRT1 in the brain tissue of infants. The results of the immunofluorescence experiment showed that compared with the sham group, the expression level of SIRT1 is significantly decreased in HI group pups, while the expression level of SIRT1 in pups pretreated with Macamide B increased significantly. The results of Western blot experiments are consistent with the results of immunofluorescence experiments. Our data indicate that Macamide B may exert a neuroprotective effect on HIBD in newborn mice by up-regulating the expression of SIRT1. Macamide B may become a new medicine effective in preventing and treating HIBD.
\end{abstract}

Keywords: Macamide B, SIRT1, Neonatal Hypoxic-ischemic Brain Damage

\section{Introduction}

Neonatal hypoxic-ischemic damage (HIBD) is brain damage caused by Perinatal hypoxia. Placental abruption, umbilical cord prolapse, amniotic fluid embolism, and uterine rupture are the common cause of mortality in HIBD [1-3]. The incidence of neonatal asphyxia is $1.5 \%(95 \% \mathrm{CI} 1.3-1.7)$, but in developed and developing countries, the incidence is $1-8 \%$ to $25 \%$ [1]. Neonatal HIBD can cause acute death and chronic mental dysfunction, even survivors will suffer from the loss of motor ability, cognitive function, and sensory function, which will cause difficulties in learning and living in the future $[4,5]$. Nowadays, the clinical treatment of HIBD mainly adopts therapeutic hypothermia [6], but few of active and effective treatments intervene and promote nerve repair. HIBD not only brings pain to the infant but also brings a burden to family and country. Consequently, it is urgent to explore effective treatment measures for HIBD.

SIRT1 is a nicotinamide adenine dinucleotide-dependent deacetylase that has been linked to longevity. It is the most abundant protein in mammalian and is involved in the regulation of many physiological functions [7]. SIRT1 participates in a variety of physiological regulation in senescence, apoptosis, transcriptional regulation, signal transduction, oxidative stress, and other important biological processes that play an important role [8]. Research shows that the sensitivity of tissues to ischemia and hypoxia in various organs should be mediated through the senescence mechanism $[9,10]$. SIRT1 is a protein that is linked 
to relieve aging whose activation can delay aging and participate in the occurrence and development of ischemia and hypoxia [11, 12]. Studies have shown that in the model of acute cerebral ischemia, SIRT1 exerts a neuroprotective effect on brain injury through mechanisms such as anti-oxidation, anti-apoptosis, and anti-inflammatory.

Maca (Lepidium meyenii Walpers), called "ginseng of South America", is an annual herb of the genus Aralia of the cruciferous family. Maca is rich in protein, vitamins, mineral elements and other nutrients, as well as secondary metabolites such as maca alkaloids, glucosides, volatile oils, sterols, polyphenols, maca alkaloids, and other secondary metabolites $10,13]$. Recently, a large number of studies have confirmed that Maca has antioxidant, anti-fatigue, enhancing fertility, regulating endocrine, enhancing immunity, anti-tumor, anti-osteoporosis, regulating blood glucose, protecting the nervous system [13]. As an effective monomer of maca, macamide $\mathrm{B}$ has been found to have important biological activities such as neuroprotection and neuromodulation [14-18], which has attracted much attention and has become a research hotspot. In the present study, the effect of Macamide $\mathrm{B}$ in the Maca plant on the expression of SIRT1 in the brain was studied and analyzed. Macamide B was found to up-regulate the expression of SIRT1 in the brain tissue of the HIBD model, suggesting that Macamide $B$ has a neuroprotective effect on neonatal HIBD. This finding has important implications for future clinical treatment.

\section{Methods}

\subsection{Establishing HIBD Model}

Preparation of HIBD model: seven-day-old neonatal mice (C57BL/6) were selected for the following steps. Mice were randomly divided into sham group, HI group and macamide B group. We chose the modified Rice-vannucci method to establish the HIBD model: the HI group, and the macamide B group were anesthetized with $3 \%$ isoflurane, and the left common carotid artery was ligated permanently in the middle of the neck, and then returned to the mother mouse to warm up and breastfeed for 1 hour. After the mice recovered, they were put into the closed space and were continuously filled with hypoxia for 4 hours $\left(8 \% \mathrm{O}_{2}+92 \% \mathrm{~N}_{2}\right)$. In the sham operation group, the left common carotid artery was exposed after anesthesia with $3 \%$ isoflurane, but the left common carotid artery was not ligated, and no hypoxia was performed.

\subsection{Immunofluorescence}

On the third day after hypoxia-ischemia, Heart perfusion was performed on pups, and brain tissue was quickly taken out and fixed in $4 \%$ paraformaldehyde for one night. The next day, the brain tissue was dehydrated and embedded in paraffin to make tissue wax. Then the tissue wax was sliced (3-5um) and the more complete brain slices were selected for immunofluorescence staining. In the process of immunofluorescence staining: first, the gradient dewaxing was carried out with xylene and ethanol, and then the antigen was repaired in $0.01 \mathrm{M}$ citric acid buffer for 20 minutes, and then sealed with goat serum at room temperature. Then anti-NeuN (1:800; Millipore, Billerica, MA, USA; cat. no. MAB377), anti-GFAP (1:800; Abcam, Cambridge, UK; cat. no. ab3670), and anti-Iba-1 (1:800; Abcam; cat. no. Ab15690) were used overnight at $4{ }^{\circ} \mathrm{C}$. On the second day, the primary antibody was aspirated, rinsed with TBS solution three times, and the corresponding secondary antibody was incubated at room temperature for 2 hours, then sealed with sheep serum, and incubated with SIRT1 antibody (1:800; Agreeableness, Cincinnati, USA) and $4^{\circ} \mathrm{C}$ at night. On the third day, the cells were incubated with the corresponding secondary antibody and stained with DAPI, and then observed under a fluorescence microscope, the localization of SIRT1 in neuronal cells, astrocytes and microglia could be found.

\subsection{Western Blotting}

After HIBD for 24 hours, the ipsilateral brain tissue was also extracted. Add a mixture of protease inhibitor (PMSF) and PIRA lysate to brain tissue for lysis. Centrifuge the brain tissue lysate in a refrigerated centrifuge at $4^{\circ} \mathrm{C}$ for $20 \mathrm{~min}(12,000 \mathrm{rpm} / \mathrm{min})$, and extract the supernatant for subsequent experiments. BCA protein quantitative kit (Beyotime Institute of Biotechnology, Shanghai) was used for quantitative analysis. Then the protein was concentrated and separated on SDS-PAGE gel, transferred to PVDF membrane (Millipore, Schwalbach, Germany), and sealed with 5\% skimmed milkforl hour. The membrane was then incubated with the primary antibody SIRT1 (1:800; Affinity, Cincinnati, USA) at $4{ }^{\circ} \mathrm{C}$ overnight. Rinse the membrane with TBST solution 3 times the next day, and then incubate the membrane with the secondary antibody for 1 hour. Finally, Bands were measured by an automatic chemiluminescence image analysis system (Tanon 5200, Shanghai, China). In this experiment, $\beta$-actin (1:800; Affinity Biosciences, China. Cat: AF7018) was employed as an internal reference protein.

\section{Results}

Macamide B can increase the expression of SIRT1 protein in brain tissue of hypoxic-ischemic newborn mice.

\subsection{Expression and Cell Location of SIRT1 in Brains of a HI Brain Injury Model}

In order to study the expression and localization of SIRT1 protein in brain tissue, we performed immunofluorescence labeling of SIRT1 protein in neuronal cells (NeuN), astrocytes (GFAP) and microglia (IBA-1) (Figure 1A). The results showed that SIRT1 was clearly labeled with neuronal cells (NeuN), astrocytes (GFAP) and microglia cells (IBA-1), indicating that SIRT1 protein was expressed in neuronal cells, astrocytes and microglia cells in the brain of HI damaged mice.

\subsection{Macamide B Up-regulated SIRT1 Protein Expression in Brains of HI Injury Model}

The expression of SIRT1 protein was quantitatively analyzed by Western blotting (Figure 2A, 2B). Western 
blotting analysis showed that the expression level of SIRT1 protein in Macamide B was higher than that in the HIBD group. Macamide B was suggested to protect against neuronal injury and increase the expression of SIRT1 protein.

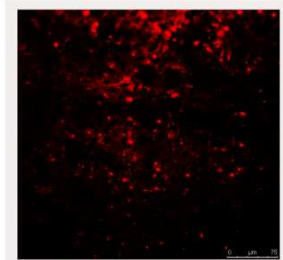

Sirt-1

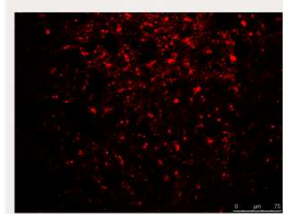

Sirt-1

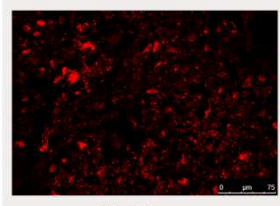

Sirt-1

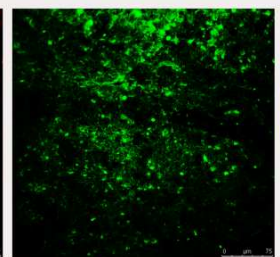

NeuN

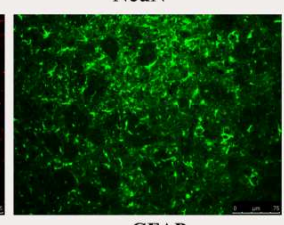

GFAP

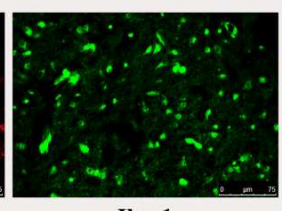

Iba-1

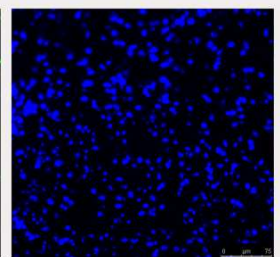

DAPI

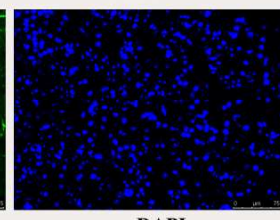

DAPI

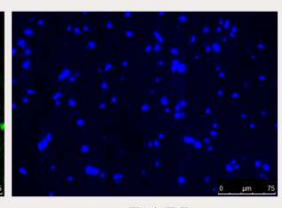

DAPI

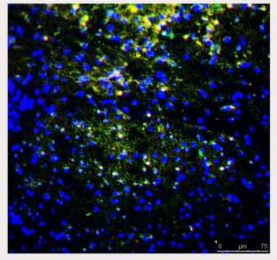

Merge

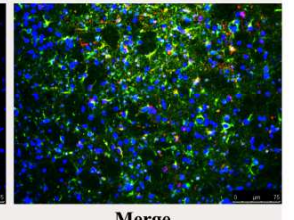

Merge

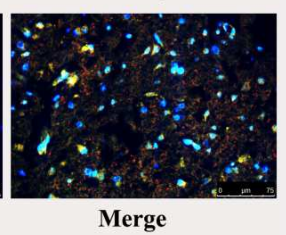

Figure 1. Cell localization of Sirt-1 in the cortex of HIBD mice. Sirt-1 was expressed in neurons (A: a-d), and astrocytes (B: a-d), and microglia (C: a-d). Bar $=250 \mathrm{um}$.

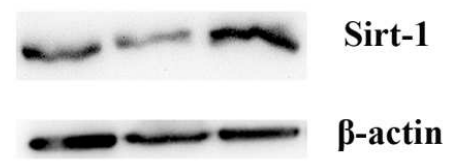

sham HI Macamide B

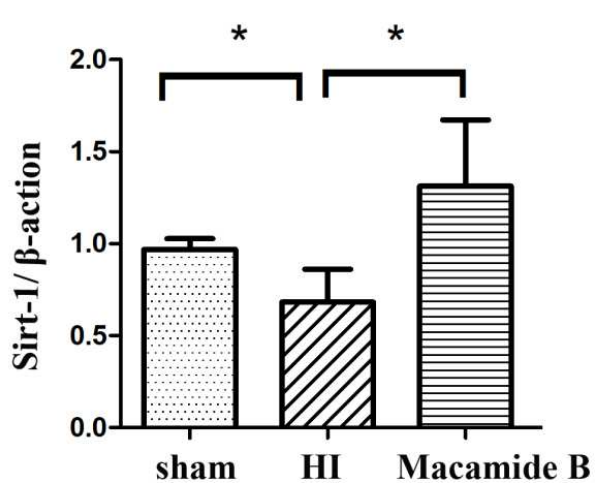

Figure 2. The expression of Sirt-1 following neonatal HIBD mice (A), Densitometric quantification of protein expression of Sirt-1 (B).

\section{Discussion}

Perinatal asphyxia is the main cause of neonatal hypoxic-ischemic encephalopathy. The mortality rate reaches $15 \%-20 \%$. About $25 \%-30 \%$ of the survivors have serious sequelae [19] which has a serious negative impact on their later life. Macamide B, as a unique chemical component in Maca, is formed by forming an amide bond between different amino compounds and various fatty acids. Although its content is low, it has significant pharmacological effects, with neuroprotective and neuromodulatory effects (analgesia, antidepressant, anti-anxiety) [14].

Sirtuins are highly conserved class III histone deacetylases that deacetylate histones and non-histone proteins in a NAD + dependent manner. SIRT1 (Silent Information Modifier) is a member of the Situin family. It is a deacetylase that relies on nicotinamide adenosine dinucleotide (NAD), which can deacetylate a variety of substrates, so it participates in the control of gene expression, metabolism and various physiological functions of aging [11, 20]. SIRT1 is mainly distributed in the human brain and spinal cord. There is evidence that SIRT1 in the brain can control neurogenesis and neuroprotection [21]. SIRT1's position in cells is not fixed because there are many kinds of cells in the body. SIRT1 can be distributed in the nucleus, cytoplasm, or both $[8,21]$. In our immunofluorescence experiments, we also proved that SIRT1 can be distributed in the nucleus, cytoplasm or both. Experiments have shown that resveratrol-mediated protection against ischemic injury requires SIRT1 activity. And resveratrol, as an effective activator of SIRT1, can protect ischemic brain tissue from energy expenditure, oxidative stress and death signal transduction [22]. In our experiment, macamide B may play a neuroprotective effect on HIBD of newborn mice by up-regulating SIRT1. The expression of SIRT1 decreased in the HI group, while the expression of SIRT1 increased significantly in the formamide B treatment group. According to the current experiments, we know that in the HIBD model, Macamide B may play a neuroprotective 
effect on HIBD in neonatal mice by increasing the expression of SIRT1 in brain tissue, thereby improving cognitive deficits.

SIRT1 has a neuroprotective function by activating a variety of targets. For example, under hypoxic conditions, SIRT1 activates hypoxia-inducible factors (HIFs) to increase the expression of erythropoietin, thereby protecting neurons from ischemia [11]. SIRT1-mediated activation of erythropoietin may be important for stimulating the formation of new blood vessels and restoring blood supply to ischemic areas. SIRT1 interacts with members of the FOXO family of transcription factors involved in regulating oxidative stress and cell death. FOXO is very important for conferring protective effects on cerebral ischemia mediated by hypoxic preconditioning. PGC1a is a promoter of mitochondrial biogenesis and an important regulator of energy homeostasis [11] The expression of SIRT1-dependent PGC1a can reduce the infarct size after middle cerebral artery occlusion and improve the nervous system score. This indicates that SIRT1 can prevent oxidative damage caused by ischemia by activating PGC1a. These downstream targets can be discussed in depth in subsequent experiments. In this experiment, we proved that macamide B may have neuroprotective effects on HIBD in neonatal mice by up-regulating the expression of SIRT1. In the future, Macamide $\mathrm{B}$ might be a promising effective drug for ameliorating brain damage in HIBD. According to the above results, we speculate that Macamide B plays a neuroprotective role through SIRT1 and downstream pathways. The Sirt1/NF-kB/Smac pathway has been shown to play vital roles in apoptosis, which is a problem worth exploring and further study [23].

\section{Conclusion}

In general, immunofluorescence staining and western blot analysis demonstrated the expression and cell location of SIRT1 in the brains of neonatal HIBD mice. (1) Through the double immunofluorescent staining, we find SIRT1 protein was expressed in neuronal cells, astrocytes and microglia cells in the brain of HI damaged mice. (2) In addition, Western Blot analysis help us demonstrated that the expression level of SIRT1 in pups pretreated with Macamide B increased significantly. This experiment showed that macamide B plays a neuroprotective role through SIRT1 and downstream pathways in newborn mice. Therefore, it can be used as a potential drug for the treatment of hypoxic-ischemic brain injury. So, mechanism underlying the regulation of the SIRT1 is a problem worth exploring and further study.

\section{Competing Interest Statement}

The authors declare that they have no competing interests.

\section{Acknowledgements}

This project was financially supported by the Guangdong Province Innovation and Entrepreneurship Training Program for University Students (grant no. 201810573045).

\section{References}

[1] Papazian O. Neonatal hypoxic-ischemic encephalopathy [J]. Medicina, 2018, 782: 36-41.

[2] Nelson KB. Lynch JK. Stroke in newborn infants [J]. The Lancet Neurology, 2004, 3 (3): 150-158.

[3] Nelson KB. Perinatal ischemic stroke [J]. Stroke, 2007, 38 (2 Suppl): 742-745.

[4] Perlman JM. Intrapartum hypoxic-ischemic cerebral injury and subsequent cerebral palsy: medicolegal issues [J]. Pediatrics, 1997, 99 (6): 851-859.

[5] Volpe JJ Neonatal encephalopathy: an inadequate term for hypoxic-ischemic encephalopathy [J]. Annals of neurology, 2012, 72 (2): 156-166.

[6] Wassink G, Davidson JO, Dhillon SK, et al. Therapeutic Hypothermia in Neonatal Hypoxic-Ischemic Encephalopathy [J]. Current neurology and neuroscience reports, 2019, 19 (2): 2.

[7] Michán S, Li Y, Chou MM, et al. SIRT1 is essential for normal cognitive function and synaptic plasticity [J]. The Journal of neuroscience, 2010, 30 (29): 9695-9707.

[8] Ramadori G, Lee CE, Bookout AL, et al. Brain SIRT1: anatomical distribution and regulation by energy availability [J]. Neurosci, 2008, 28 (40): 9989-9996.

[9] Meng X, Tan J, Li M, et al. Sirt1: Role Under the Condition of Ischemia/Hypoxia [J]. Cellular and molecular neurobiology, 2017, 37 (1): 17-28.

[10] Muhammad I, Zhao J, Dunbar DC, et al. Constituents of Lepidium meyenii 'maca' [J]. Phytochemistry, 2002, 59 (1): 105-110.

[11] Morris KC, Lin HW, Thompson JW, et al. Pathways for ischemic cytoprotection: role of sirtuins in caloric restriction [J], Cereb Blood Flow Metab, 2011, 31 (4): 1003-1019.

[12] Gan L, Mucke L. Paths of convergence: sirtuins in aging and neurodegeneration [J]. Neuron, 2008, 58 (1): 10-14.

[13] Gonzales GF. Ethnobiology and Ethnopharmacology of Lepidium meyenii (Maca), a Plant from the Peruvian Highlands [J]. Evidence-based complementary and alternative medicine, 2012, 2012: 193496.

[14] Piacente S, Carbone V, Plaza A, et al. Investigation of the tuber constituents of maca (Lepidium meyenii Walp.) [J]. Journal of agricultural and food chemistry, 2002, 50 (20): 5621-5625.

[15] Yali Wang YW, Brian McNeil, Linda M, et al. Harvey, Maca: An Andean crop with multi-pharmacological functions [J]. Food Research International, 2007, 40 (7): 783-792.

[16] Ganzera M, Zhao J, Muhammad I, et al. Chemical profiling and standardization of Lepidium meyenii (Maca) by reversed phase high performance liquid chromatography [J]. Chemical \& pharmaceutical bulletin, 2002, 50 (7): 988-991.

[17] Uchiyama F, Jikyo T, Takeda R, et al. Lepidium meyenii (Maca) enhances the serum levels of luteinising hormone in female rats [J]. Journal of ethnopharmacology, 2014, 151 (2): 897-902. 
[18] McCollom MM, Villinski JR, McPhail KL, et al. Analysis of macamides in samples of Maca (Lepidium meyenii) by HPLC-UV-MS/MS [J]. Phytochemical analysis: PCA, 2005, 16 (6): 463-469.

[19] Jiang W, Guo M, Gong M, et al. Vitamin A bio-modulates apoptosis via the mitochondrial pathway after hypoxic-ischemic brain damage [J]. Molecular brain, 2018, 11 (1): 14.

[20] Rahman S, Islam R Mammalian. Sirt1: insights on its biological functions $[\mathrm{J}]$. Cell communication and signaling: CCS, 2011, 9: 11.
[21] Zakhary SM, Ayubcha D, Dileo JN, et al. Distribution analysis of deacetylase SIRT1 in rodent and human nervous systems [J]. Anatomical record (Hoboken, NJ: 2007), 2010, 293 (6): 1024-1032.

[22] Howitz KT, Bitterman KJ, Cohen HY, et al. Small molecule activators of sirtuins extend Saccharomyces cerevisiae lifespan [J]. Nature, 2003, 425 (6954): 191-196.

[23] Ji K, Sun X, Liu Y, et al. Regulation of Apoptosis and Radiation Sensitization in Lung Cancer Cells via the Sirt1/NF-kB/Smac Pathway [J]. Cell Physiol Biochem, 2018, 48 (1): 304-316. 